\title{
Let's get critical
}

\author{
Derek Richards \\ Editor, Evidence-based Dentistry \\ Evidence-Based Dentistry (2005) 6, 85. doi:10.1038/sj.ebd.6400364
}

One of the key skills needed for evidencebased practice is the ability to critically appraise information. Unfortunately, it is a skill (that is) not taught routinely at undergraduate level, but increasingly it is happening at postgraduate level. Initiatives such as the inclusion of a critical reading paper in the Faculty of General Dental Practitioners UK Membership examination has been a driver to encourage critical appraisal, but sadly, exposure of general dental practitioners to courses and workshops in critical appraisal skills has been limited. Courses in critical appraisal are available in the UK, but few are directed solely towards dental practitioners, and those that do target dental practioners tend to address wider topics of evidence-based practice or systematic reviewing (see events page). There are also several books and tools available (www.phru.nhs.uk/casp/critical_appraisal_tools.htm) and we review one such interactive workbook and CD-ROM in this issue. However as with many things the more you do it the easier it becomes.

The ability to discriminate between good research, poor research and downright misleading research is increasingly important as information of all sorts is increasingly available on the internet. The widespread availability of internet access has for example, led to an enormous increase in the numbers of searches being carried out on PubMed (www. ncbi.nlm.nih.gov/entrez/query.fcgi?DB= pubmed a service provided free by the
American National Library of Medicine that includes over 15 million citations from MEDLINE and other life science journals for biomedical articles back to the 1950s), with numbers increasing from 163000 in January 1997 to 68080000 in March 2005.

A common finding by those conducting systematic reviews is that not enough information is provided in the original papers to enable synthesis of the data into meta-analysis, even when the research question being addressed by the paper is the same one the systematic reviewers are looking at. This is frustrating for a number of reasons; there are now well-recognised standards for the reporting of a range of study designs, ${ }^{1}$ which are not followed. Most major journals have in place systems of peer review and to my mind one of the roles of peer reviewers and journal editors should be to require authors to follow these standards. One of the roles of journals is to disseminate research. In order to do this effectively and perhaps influence the uptake of clinically relevant research in practice, the research should be understandable to the audience. Here I believe that the readership of the journal has a role, for if research is not understandable or if relevant information is missing, the readership should complain. Preferably, this should be in a structured critical fashion as is taught as one of the key skills of evidence-based practice. This sort of structured criticism will have an effect on scientific publica- tion if the clamour is strong enough, but the temptation for out and out criticism should be resisted for as Dale Carnegie (1888-1955) pointed out

"Any fool can criticize, condemn, and complain - and most fools do."

One organisation that does have a very robust and systematic process of review and appraisal is the Cochrane Collaboration, and next year will see the 10th anniversary of the Cochrane Oral Health Group's editorial base being in Manchester; the Group having been initially established in America in 1994. To celebrate the 10th anniversary the Oral Health Group will be hosting a symposium in Manchester on 30-31 May 2006 on 'Incorporating evidence into dental practice'. Details of the site will appear of the Group's website (www.cochrane-oral. man.ac.uk/) which has had a major overhaul recently and is worth a visit anyway.

The growth of the Oral Health Group over the past ten years has been impressive, with 51 reviews and 51 protocols published in The Cochrane Library, and a further 58 titles registered with the Group. The Oral Health Group Trials Register has grown substantially from 3500 references relating to trials in 1997 to currently over 20000 . It is an invaluable resource for all those wanting to undertake a systematic review.

1. Niederman R, Richards D, Matthews D, Shugars D Worthington $\mathrm{H}$, Shaw W. International standards for clinical trial conduct and reporting. J Dent Res 2003; 82:415-416. 